\title{
Museos, memoria y antropología a los dos lados del Atlántico. Crisis institucional, construcción nacional y memoria de la colonización
}

\author{
por \\ Jesús Bustamante ${ }^{1}$ \\ Instituto de Historia, CCHS-CSIC
}

\begin{abstract}
Los museos de contenidos antropológicos y/o que se refieren a espacios de colonización occidental atraviesan una larga crisis que está generando la renovación general de las instituciones más antiguas, el desarrollo de nuevos modelos museológicos y el surgimiento de propuestas críticas y expositivas muy variadas. Se trata de un fenómeno que afecta sobre todo a la Antropología como disciplina científica, pero también y de forma cada vez más importante a la Historia y Bellas Artes. Este trabajo intenta ofrecer una visión de conjunto basándose en la comparación de los procesos que se están produciendo tanto a un lado como al otro del Atlántico, y subrayando la importancia de los casos latinoamericanos por su variedad, originalidad y por la complejidad de situaciones socio-culturales a los que van asociados.
\end{abstract}

Palabras Clave: Museos; memoria; antropología; colonización; construcción nacional; patrimonio; comunidad; modelos museológicos.

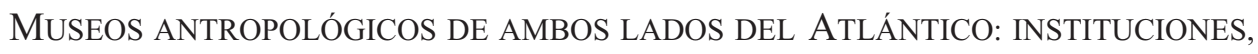
DISCIPLINAS Y CRISIS

La apertura en el año 2006 del nuevo Musée du quai Branly, así sin otro nombre específico, pero con varios subtítulos: «là où dialoguent les cultures» y «un musée pour les artes non occidentaux» (según su página web²), repre-

1 Este trabajo se inscribe en el proyecto de investigación «Museos, memoria y antropología: América y otros espacios de colonización», financiado por el Ministerio de Ciencia e Innovación del Gobierno de España (referencia: HAR2009-10107).

2 Véase http://www.quaibranly.fr/fr./l-etablissement-public/index.html que corresponde a la «Présentation» de la institución («L’Établissement public»). 
senta la culminación de un largo proceso de redefinición de todo un grupo de museos franceses de contenidos antropológicos, pero sobre todo implicó la refundición y refundación de dos notables museos parisinos: el Musée de l'homme y el Musée des arts d'Afrique et d'Océanie. Uno era el museo antropológico por antonomasia (no solo en Francia), y el otro, tal como expresaba la denominación con la que fue fundado en 1931, era el Musée des colonies. El largo proceso de reconversión de ambos museos, que a partir del año 2000 utilizaron al propio Musée du Louvre y su Pavillon des Sessions como plataforma de tránsito y de ensayo, ha sido objeto - y sigue siéndolo- de todo tipo de debates en ámbitos que exceden el meramente académico ${ }^{3}$. El nuevo museo resultante es hoy un referente obligado - positivo o negativo - en el complejo proceso de transformación que desde finales del siglo pasado está afectando de forma generalizada a los museos cuyos contenidos tienen un carácter antropológico y/o se refieren a espacios de colonización occidental. Un fenómeno de notable importancia y de consecuencias — culturales y políticas - que no son precisamente neutras, sobre todo desde una perspectiva latinoamericana.

En Europa, además del caso parisino que acabamos de mencionar, este proceso ha implicado la desaparición del Mankind Museum de Londres, «reintegrado» en 1997 al British Museum y a su clásico — pero más bien atestadoedificio de Bloomsbury ${ }^{4}$. Y también a este mismo proceso responde la profunda reestructuración realizada en el Troppenmuseum (antiguo Koloniaal Museum) de Ámsterdam, la que se está llevando a cabo ahora mismo en el Linden-Museum de Stuttgart (Staatliches Museum für Völkerkunde) o la que se va a iniciar en un futuro inmediato en el Musée Royal de l'Afrique Centrale de Tervuren (antiguo Musée du Congo, junto a Bruselas, de fuerte sabor colonial), entre otros muchos casos.

Se trata de un fenómeno que encontramos igualmente en Estados Unidos, como demuestran los complicados debates surgidos en torno a la creación del

3 La bibliografía generada por estos acontecimientos ha sido muy amplia, me permito destacar Dupaigne, 2006, que hace una exposición fuertemente crítica; L'Etoile, 2007, que aprovecha la situación de conflicto para hacer una «antropología de los museos de antropología» en Francia; y Price, 2007, que intenta hacer una exposición ecuánime de los acontecimientos y de las distintas tendencias.

${ }^{4}$ El llamado Museum of Mankind era propiamente el Departamento de Etnología del Museo Británico, que entre 1970 y 1997 contó con notable autonomía además de un edificio propio. Pero conviene recordar el carácter especialmente conservador de las instituciones británicas, como demuestra el hecho de que el Bristih Museum of Natural History de Londres fue creado en 1881-83 pero solo en 1963 hubo una separación legal completa del British Museum propiamente dicho. Por cierto, lo que entre 1970 y 1997 se denominaba Department of Ethnography, hoy lleva por nombre Department of Africa, Oceania and the Americas. 
National Museum of the American Indian en 1989, como consecuencia de la incorporación a la Smithsonian Institution de Washington del antiguo Museum of the American Indian (institución hasta entonces privada, creada por Gustave Heye en 1916 y cuya sede central estaba en Nueva York). O como atestiguan de forma todavía más clara - dentro de la misma Smithsonian Institution - las agrias polémicas (suscitadas sobre todo por afroamericanos) que llevaron a que el National Museum of Natural History de Washington clausurara en 1991 sus salas de etnografía africana y se decidiera crear un National Museum of African American History and Culture en 20035. Se trata de un conjunto de debates que, en primer lugar, se ha centrado en la naturaleza y legitimidad de los fondos mismos de estos museos, lo que ha llevado a promulgar el Native American Graves Protection and Repatriation Act y leyes anexas, y a crear una Repatriation Office en la propia Smithsonian Institution ${ }^{6}$. Pero que, en segundo lugar, también han puesto en cuestión la selección de piezas en exposición, los discursos expositivos utilizados y hasta los públicos a los que van dirigidos $^{7}$. Pero como consecuencia de todo ello, hoy no es raro encontrar textos de visitantes y estudiosos denunciando que los nuevos museos - $\mathrm{y}$ especialmente el National Museum of the American Indian - están ahora excesivamente inclinados al punto de vista indígena y que intentan ofrecer una visión homogenizadora - basada con frecuencia en clichés románticos - de un universo caracterizado por una diversidad inmensa, lleno además de tensiones y conflictos (lo que hace muy difícil coordinar contenidos, hacer propuestas que lo abarquen en su plenitud, y más aún olvidar la dimensión política) ${ }^{8}$. En un notable artículo aparecido en The New York Times de enero de 2011, Kate Taylor llega a poner el museo sobre el indio americano como ejemplo a evitar en el diseño del nuevo museo sobre la historia y cultura de los afroamericanos ${ }^{9}$. Una dura crítica que remite explícitamente a las dos cuestiones básicas que

5 El edificio de este nuevo museo debería empezar a construirse en 2012 y finalizarse en 2015. Mientras, ocupa una galería en la segunda planta del National Museum of American History. Véase su página web: http://nmaahc.si.edu/section/about_us.

6 Véase la página web de la Repatriation Office con todo tipo de información al respecto: http://anthropology.si.edu/repatriation/index.htm.

7 Véase West et al., 2000. Cooper, 2008.

8 Véase Rothstein, 21 septiembre 2004. Véase además Lonetree y Amanda (eds.), 2008.

9 «The newest Smithsonian museum, the National Museum of the American Indian, has been criticized as being overly reverential and lacking in historical perspective.... Reluctant to impose a narrative written by white anthropologists and historians, the Indian museum largely rejected outside professional scholarship. Its exhibitions, which speak mainly in an American Indian voice, have been criticized as incoherent and plagued by romantic cliché» (Taylor, 22 enero 2011). 
todo museo debe responder — «What story will it tell?» $\mathrm{y}$ «who will be listening?»—- con el importante añadido de que además se trata de «museos nacionales», es decir, un tipo de instituciones que «bears the burden of being the "official" - that is, the government's version of the... history", y que se financian con dinero público, o sea de todos (no de un grupo, etnia o clase).

Este conjunto de hechos, críticas y contracríticas que están afectando $-\mathrm{y}$ renovando - tan profundamente a los museos de antropología en todas sus variantes, tiene bastante que ver con lo que podríamos llamar un problema «de origen». Es bien sabido que el surgimiento y expansión de los museos antropológicos especializados se produjo en la segunda mitad del siglo XIX, como una rama más de la institucionalización museística que se encontraba entonces en pleno florecimiento ${ }^{10}$. También es bien conocido que esta «edad de oro» de los museos, como suele denominarse al periodo, se produjo en estrecha relación con otros dos fenómenos: el surgimiento y consolidación de los estados nacionales, y el surgimiento e institucionalización de las principales ciencias modernas (que, en Occidente, alcanzaron entonces un reconocimiento social que nunca antes habían tenido). Entre ellas, claro está, la Antropología y ciencias - y pseudociencias - afines.

Entre museos, ciencias y estado-nacional existe una estrecha relación que alcanza su máxima expresión en los llamados «museos nacionales». Instituciones entendidas como centros de conservación y estudio, pero también como lugares de educación de la ciudadanía, con unas prácticas y un discurso expositivo que necesariamente responden a condicionamientos y fines políticos (y no solo científicos). Se trata de los grandes lugares de la memoria, allí donde el discurso expositivo intenta expresar cómo una sociedad quiere ser vista y cómo ve o quiere ver a las demás, en el presente y en su pasado. Lo que evidentemente hace necesario revisar ese discurso expositivo cada cierto tiempo.

En el caso de los museos cuyo foco de atención son los seres humanos y los espacios de expansión de la sociedad occidental por otros continentes (desde el punto de vista europeo) o por el propio territorio continental (desde el punto de vista americano, tanto del hemisferio norte como del sur), la relación entre museos, ciencias y estado-nacional no solo se ha vuelto cada vez más problemática, sino que está llena de tensiones y contradicciones que varían considerablemente según el punto de vista que el estudioso asuma.

En primer lugar, el desarrollo mismo de este tipo de museos a lo largo de los dos últimos siglos (XIX y XX) es notablemente diferente según el lado del Atlántico en que nos ubiquemos. En suelo europeo son instituciones que nacen

10 Sobre esta cuestión, véase Bustamante, en prensa. 
sobre todo como escaparates del colonialismo, basadas en el valor de lo diferente y exótico, y se dedican al estudio, conservación y difusión de materiales pertenecientes a culturas de todo el mundo (además de las culturas tradicionales propias). Por el contrario, en suelo americano, y especialmente en América Latina, se asocian desde el principio a lo que es propio, a lo que es local y su objetivo es todo aquello que define o puede definir la identidad de los estados nacidos tras las independencias. Las instituciones que en suelo europeo vendrían a configurar la imagen del «otro», en suelo americano vienen a configurar la «propia imagen» o una parte importante - fundacional- de ella. Se trata de un fenómeno especialmente significativo y de notables consecuencias teóricas, porque europeos y americanos elaboran sus discursos a partir de los mismos presupuestos científicos y lo hacen en instituciones que se reconocen mutuamente como homólogas, compartiendo los mismos espacios y códigos académicos de debate, legitimación, publicación, etc.

En segundo lugar, los procesos de descolonización que culminaron en la década de 1970 han generalizado — quizá deberíamos decir «globalizado»—a todos los antiguos espacios coloniales esa contradicción que antes hemos descrito como característica de las instituciones museísticas a un lado y otro del Atlántico. Además han vuelto obsoletos - y políticamente muy incorrectoslos antiguos «museos coloniales» (vigentes hasta la década de 1960). Pero es que, como daño colateral, también han dejado en una situación muy problemática a los museos antropológicos en general y hasta a la Antropología como disciplina. A ello se ha agregado, en los últimos años, la crisis del estado-nacional en el contexto de la globalización, que ha provocado entre otras cosas el debilitamiento - y hasta la pérdida — del discurso que venía rigiendo las instituciones museísticas desde el siglo XIX, como formadoras de ciudadanía, y ha modificado la actitud de la sociedad mayoritaria frente a los distintos grupos étnicos y/o minoritarios.

Sobre estos planteamientos se ha desarrollado el proyecto de investigación cuyos primeros resultados exponemos aquí, concentrando las cuestiones generales en este artículo, al que siguen después varias monografías sobre casos de estudio específicos.

\section{MuSEOS ANTROPOLÓGICOS: CULTURALISTAS, HISTORIZANTES, ESTETIZANTES}

Antes hemos comentado que la contrastación entre los casos europeos y latinoamericanos descubre, en primer lugar, algo bastante evidente: que los museos antropológicos — según el continente - se fundamentan en dos tipos distintos de memoria. Para los europeos se trata de una memoria colonizadora y 
de expansión (expresada en la recolección de objetos que se categorizan como exóticos). Para los americanos se trata de una memoria nacional, en la que se combina la condición de colonizado - frente a Europa - con la de colonizador - frente a las poblaciones indígenas y territorios propios (expresadas en la recolección de objetos que se categorizan como autóctonos en su sentido literal). Todo lo cual implica, naturalmente, políticas y discursos diferenciados.

Pero en segundo lugar la comparación nos permite descubrir un segundo nivel de diferenciación no tan evidente en primera instancia y que se refiere a una definición de «antropológico» y «colonial» que es distinta según los continentes. En Europa los museos «coloniales» se entienden sobre todo como «antropológicos» y van unidos a un periodo de la propia historia que hoy resulta mejor no enfatizar, e incluso olvidar. En América (y también en otros continentes) esos mismos museos se entienden sobre todo como «históricos» y se asocian a un periodo muy preciso de la propia historia (la que suele llamarse «virreinal» o «colonial»), que resulta poco deseable pero que es preciso rememorar como denuncia y conservar su riqueza patrimonial, al menos por su valor artístico. A su vez, los museos «antropológicos»-los mismos que en Europa están lastrados por su asociación con lo colonial- en América se entienden como «ahistóricos» y remiten a las esencias eternas de la patria y de la nación, en el pasado y en el presente, lo que les otorga una especial importancia y significación política, como ejemplifica de forma clarísima el Museo Nacional de Antropología de México ${ }^{11}$.

En tercer lugar la comparación de casos permite proponer tres categorías básicas a la hora de clasificar los distintos museos de tipo antropológico y sus discursos expositivos:

«Culturalistas», que enfatizan especialmente la dimensión cultural, lo que puede hacerse subrayando las interactuaciones y por tanto las tensiones (una práctica que suele ser puntual y estar asociada a procesos reivindicativos), o mostrando un universo "aproblemático» y atemporal a modo de un "archipiélago» de culturas con poca o ninguna interacción entre ellas ${ }^{12}$.

«Historizantes», que enfatizan procesos sociales ocurridos en un periodo de tiempo, subrayando las interacciones y los conflictos, y mostrando especialmente sus consecuencias vistas desde el presente. Tradicionalmente este tipo de museos suelen abarcar periodos muy largos y proponen un modelo evolutivo cultural explícito asociado a un sujeto (la nación, un grupo étnico o racial, la humanidad); aunque hoy también los encontramos con un carácter li-

11 Sobre esto, véase Bustamante, 2005.

12 Véase Fabian, 1983 (de donde tomo además la imagen de «archipiélago»). Pero véase también Fabian, 2007, en que «revisita» los mismos temas casi veinticinco años después. 
teralmente histórico, es decir, que se centran en un periodo y lugar específicos, como el momento de la ocupación colonial de un territorio, con carácter más bien crítico y omitiendo consecuencias generalistas.

«Estetizantes», que se centran en los objetos en sí mismos y en su propia capacidad expresiva, omitiendo toda referencia a tensiones culturalistas, históricas o políticas en general. Probablemente es el modelo más actual, el que está de moda y parece responder mejor a las tendencias del momento, con una evidente fascinación por los objetos bien diseñados, bien acabados, a pesar de su clara condición fragmentaria y, sobre todo, sin que de ello se desprenda ninguna inquietud, ninguna consecuencia social o moral. Algo así como ocurre con el universo de la propaganda comercial más común.

Por la experiencia acumulada hasta ahora, se trata de los tres modelos expositivos que se utilizan con más frecuencia en este tipo de museos. El culturalista suele ser el habitual de los museos antropológicos universitarios (como el Museum Volkenkunde de Leiden o el Phoebe A. Hearst Museum of Anthropology de la Universidad de Berkeley) y, aunque tradicionalmente ha venido ajustándose al paradigma del «archipiélago» de culturas, hoy no es raro que incorporen además propuestas que intentan aportar una visión algo más integrada y universalista (para lo que recurren a temas «transversales» como, por ejemplo, los usos del calzado o de la cerveza en las diferentes culturas del mundo, en el presente y en el pasado; ejemplo este último, por cierto, que tomo directamente del caso de Berkeley). Un rasgo típico de este modelo es que con frecuencia se pretende reducir el valor de las piezas expuestas a su estricta dimensión cultural primaria, obviando todo lo demás, especialmente todo lo que la hace excepcional o extraordinaria, como lo que va asociado a su belleza formal, riqueza material, antigüedad, historia conocida, características de sus poseedores antiguos y usos que le dieron, etc. Lo que con frecuencia es una notable simplificación y obliga a pasar por alto demasiadas cosas. Consecuencia de ello es que pueden ponerse juntos y al mismo nivel de significación cultural un antiguo recipiente egipcio o maya para líquidos fermentados con una lata de cerveza moderna, al margen de lo diferente que pueda ser su antigüedad, elaboración y riqueza artística, al margen también de que respondan a modelos económicos y productivos muy distintos, y sin tener en cuenta el abismo que sin duda existía en los usos de los embriagantes entre esas tres culturas, por ejemplo. Pero esta es una tendencia muy difundida, buena parte de cuyo éxito radica precisamente en su tendencia simplificadora, que parece responder además a las presiones que existen sobre las instituciones museísticas para que actúen como centros de entretenimiento dirigidos a un público cada vez más joven. 
Por su parte el modelo historizante, sobre todo en su versión de gran discurso evolutivo-cultural (casi siempre asociado a una comunidad o a una nación específica) ha desaparecido casi por completo de los museos que tenían un origen colonial (como el Tropenmuseum de Ámsterdam, que lo ha sustituido por una exposición culturalista con una importante sección histórica, en su sentido literal y en su dimensión más crítica). Sin embargo, el gran discurso evolutivo-cultural, que es de hecho con el que se constituyeron la mayor parte de los grandes museos nacionales de antropología, como el antiguo Musée de l’homme sin ir más lejos, sigue presente de forma más o menos explícita en multitud de museos locales y de comunidad latinoamericanos (como demuestran varios de los trabajos incluidos en este mismo número monográfico de Revista de Indias, pero véase especialmente el de Mónica Quijada), además de en algunos museos nacionales (como el Museo Nacional de Antropología de México, que sigue manteniendo la propuesta expositiva con la que esta institución se fundó en 1964, con esa imponente y muy didáctica secuencia de salas que va de los primeros pobladores y el Formativo hasta los «Aztecas», la famosa «sala número 7», la mayor del edificio y clara culminación simbólica de todo el museo hasta por su diseño arquitectónico).

En cuanto al modelo estetizante cabe repetir que es sin duda una propuesta en alza, a la que se está recurriendo cada vez más tanto a un lado como al otro del Atlántico, a la hora de «resolver»o al menos neutralizar las graves tensiones y contradicciones que la temática étnica y antropológica provoca cuando va asociada a lo colonial y/o a la construcción del estado-nacional. El Musée du quai Branly de París ejemplifica muy bien este modelo y probablemente sea el caso más conocido (véase la reflexión sobre este tema y sus profundas raíces en el trabajo de Christine Laurière en este mismo número monográfico), pero hay muchos otros que además son muy variados en sus características, entre ellos, uno tan difícil de clasificar como el de Arte Precolombino y del Vidrio Moderno de Antigua Guatemala, y yo agregaría también el Museo Nacional del Virreinato de Tepozotlán, México, con su singular acotamiento y exposición de lo colonial que lo convierte en un declarado museo de Bellas Artes (véanse los correspondientes trabajos de Marta Casáus y de Tomás Pérez Vejo en este mismo número de la revista).

Por cierto, este tipo de museos que intentan evadir la problemática social y política por medio de una solución estetizante, no debe confundirse con otro tipo de museos que surgieron como consecuencia de la pasión recolectora de artistas y coleccionistas fascinados por el atractivo formal de un tipo singular de piezas que se juzgaban exclusivamente desde la óptica occidental del momento, sin importar su origen cultural o temporal. Este tipo de museos - por lo general, bellísimos - tienen una clara vocación de vanguardia estética, pero 
solo secundariamente tienen una dimensión antropológica en sentido estricto (suelen recoger piezas de gran valor por su calidad estética o su originalidad, pero no por su poder de contextualización ni de representación cultural), como el De Young de San Francisco, el Rufino Tamayo de Oaxaca o el Museo delle Culture Extraeuropee (Collezione Serge e Graziella Brignoni) de Lugano, por poner tres ejemplos muy diferentes, pero que han surgido de un fenómeno colector muy similar.

Los tres modelos expositivos que venimos comentando tratan de responder una cuestión que es común a los museos históricos y antropológicos, pero que se hace especialmente aguda en estos últimos: la del «sujeto» y «objeto» museístico. Es decir, quién es el que hace el museo y formula su discurso expositivo. Y quién o quiénes son los que ejercen de objetos pacientes sobre los que se construye ese museo, con todos sus discursos. Y a estas dos cuestiones iniciales es preciso agregar una tercera no menos importante: quién es el público al que se dirige la institución y sus programas expositivos, o más exactamente: ese público debe identificarse con el quién-sujeto o con el quién-objeto. La cuestión es especialmente seria porque debe tenerse en cuenta que, en el caso de los museos antropológicos, la noción de ser humano como objeto de museo debe entenderse de un modo literal, que se materializa físicamente en colecciones que con frecuencia pueden ser muy ambiguas e inquietantes desde un punto de vista ético (cuando no directamente inaceptables desde una perspectiva actual). Lo cual no es precisamente un problema menor para este tipo de instituciones, aunque no es el único ni el más difícil.

Llegamos así a las tres grandes cuestiones o problemas que afectan hoy a todos los museos y, desde luego, a los antropológicos:

Patrimonio. Una cuestión que gira especialmente sobre el tema de a quién pertenecen los objetos patrimoniales, optando entre quien los produce o quien los guarda, quien ha generado los objetos culturales en su materialidad o quien los ha transformado en piezas patrimoniales propiamente dichas, otorgándoles para ello un cierto tipo de significaciones y preservándolos materialmente. Debe añadirse que en el concepto de patrimonio interviene además un principio de territorialidad muy complejo, asociado a lo «nacional», que define lo patrimonial como una riqueza inajenable del propio suelo, como si fuera un «producto natural» del mismo (al modo de los metales preciosos, el petróleo o cualquier otra materia prima), cuyo propietario fuera indiscutiblemente la actual sociedad nacional que ocupa ese espacio territorial, al margen del devenir del tiempo y de los cambios en las sociedades humanas en general, y de los que se han producido en ese territorio en particular.

Comunidad. Se trata de una noción clave, pero muy confusa, que está en estrecha relación con la cuestión anterior. Puede referirse tanto a la comunidad 
propietaria de ese patrimonio en la actualidad, como a la que lo ha realizado materialmente o, incluso, a la que se siente representada por esos objetos patrimoniales aunque tenga poco o nada que ver con la comunidad cultural originaria (pero con la que se identifica o de la que se siente heredera por compartir, por ejemplo, el mismo espacio geográfico de origen). Sin embargo, el concepto de comunidad también se puede aplicar al grupo social al que se dirige la institución museística o dentro del cual se ha edificado (cualquiera que sea la relación que guarde con los fondos patrimoniales).

El museo mismo y las funciones que se supone debe desempeñar como institución. Funciones que en la actualidad se han incrementado considerablemente sobre las atribuciones clásicas, y abarcan desde la conservación del patrimonio, la investigación científica y la difusión del conocimiento, pasando por la formación cívica de la ciudadanía o la de actuar como referente simbólico para ciertos grupos sociales, hasta la de actuar como centros de entretenimiento y de otros servicios comunitarios - dentro de la llamada «industria cultural del ocio»- para mayores y la infancia, por ejemplo.

Este complejísimo cuadro, que aquí nos limitamos a esbozar mínimamente, es el que se desarrolla en las sucesivas contribuciones reunidas en este número monográfico, cada una centrada en una problemática distinta, tratando de afrontar su complejidad y su diversidad. Museos nacionales, museos privados, museos comunitarios, patrimonio y patrimonialización, gran discurso cívico y discurso alternativo, conflictos funcionales y museología subalterna, museos de frontera, de coleccionistas y universitarios... Una temática inagotable, para cuyo estudio las experiencias latinoamericanas son especialmente estratégicas, que es necesario asumir como propia de las Ciencias Humanas y Sociales, y que en esta contribución específica se afronta desde la perspectiva puramente antropológica.

\section{EL YO, LOS OTROS, LOS MUSEOS Y DÓNDE QUEDA LA ANTROPOLOGÍA}

Benoît de l'Etoile, en su monografía sobre los museos antropológicos en Francia ${ }^{13}$, que él ofrece como una «anthropologie du musée», afirma que desde las viejas exposiciones coloniales, pasando por el primer museo del Trocadero y terminando en el actual Quai Branly, dedicado «à l'art et aux cultures des civilisation non occidentales» (según Germain Viatte, director de su proyecto museológico), todos ellos se definirían por contraposición a lo que es

13 L'Etoile, 2007: 11. 
occidental y, de forma implícita, contemporáneo (aquí pesa, evidentemente, la poderosa propuesta analítica de Johannes Fabian y el papel de lo temporal en la definición del objeto antropológico tradicional) ${ }^{14}$. Es decir, todos ellos corresponderían a la categoría genérica de «un musée des Autres».

A partir de este punto, L'Etoile hace una propuesta generalizadora contundente: «Se puede... repartir los museos, muy especialmente los de antropología y los de historia, en dos categorías desde el punto de vista de su relación con la identidad: museos de uno mismo y museos de los otros $\rangle^{15}$. Reparto que no parece muy equilibrado en cuanto a su distribución porque: «la situación... nornal, y con mucho la más frecuente, es la del "museo de uno mismo" que expone los tesoros de un grupo local, de una comunidad $\rangle^{16}$.

El principio de «comunidad», que — como ya se ha comentado- es bastante complicado y ha sido objeto de numerosos trabajos en su relación con los museos ${ }^{17}$, está explícitamente tomado por L'Etoile de Claudio Lomnitz, para quien «los museos de historia y de arte son instituciones que concentran los bienes inalienables de una comunidad (habitualmente un pueblo o una nación) y los ordenan de forma tal que constituyen una visión del presente de esa comunidad a partir de su pasado, por oposición al pasado y al presente de otras comunidades ${ }^{18}$.

Para Benoit de l'Etoile esa definición correspondería de forma canónica a lo que él denomina «museos de uno mismo» (musée de Soi), un tipo de institución a la que atribuye como función principal responder a la cuestión del «Quiénes somos» y hacerlo pensando tanto en el visitante extranjero como en la propia «comunidad» que «el museo tiene como objetivo fortalecer o constituir» ${ }^{19}$.

El problema es que esta definición se ajusta fácilmente a una notable cantidad de instituciones, muy variadas desde cualquier otro punto de vista. Tal

14 Véase Fabian, 1983. Se trata de un tema al que este autor ha vuelto en varias ocasiones, véase muy especialmente Fabian, 2001 y 2007.

15 «On peut... répartir les musées, et tout particulièrement les musées d'anthropologie et d'histoire, en deux catégories du point de vue de leur rapport à l'identité: les musées de Soi et les musées des Autres» (L'Etoile, 2007: 11). Todas las traducciones son mías.

16 «La situation... normale, et de loin la plus fréquente, est celle d'un "musée de Soi" qui expose les trésors d'un groupe local, d'une communauté» (L'Etoile, 2007: 11).

17 Sobre este tema, véase por ejemplo Karp, Kreamer y Lavine (eds.) 1992, así como Peers y Brown (eds.), 2003.

18 Lomnitz, 1999: 112. Pero véase todo el capítulo que lleva por título: «Dos propuestas para los museos del futuro».

19 «En s'adressant à la fois au visiteur extérieur et à la "communauté" elle-même, que le musée vise d'ailleurs souvent à renforcer, voire à constituer» (L'Etoile, 2007: 12). 
como se está utilizando en este contexto, el concepto de «comunidad» no remite a un grupo social o étnico más o menos minoritario, como ocurre cuando hablamos, por ejemplo, de museos propiamente comunitarios (véase el artículo de Manuel Burón en esta misma revista), sino que más bien se refiere a la «sociedad» mayoritaria misma, ya sea en su forma más amplia como «pueblo» o «nación», o ya en una modalidad más reducida como un grupo regional que, sin embargo, se identifica o se define en términos equivalentes. Un concepto de comunidad a gran escala, podríamos decir.

L'Etoile ejemplifica su propuesta con casos tan diversos como el Musée Alsacien (Strasbourg), el Ecomusée de la Grande Lande de Marquèze (Landes de Gascogne) o incluso el Museo Archeologico Nazionale de Nápoles, que, junto a una evidente ruptura con el pasado romano, también quiere mostrar continuidades de prácticas cotidianas y técnicas (como las asociadas al pan o a las olivas), casi siempre vinculadas al suelo y a la tierra, que hacen de ese pasado algo a la vez lejano y familiar. De hecho todos esos museos tendrían en común ser «herederos de proyectos de afirmación de una identidad colectiva enraizada en un pasado común $»^{20}$. Todos remitirían a un «nosotros encarnado en el museo por los diversos objetos que dan testimonio del pasado de la comunidad en cuestión y que, normalmente, provienen de su territorio» ${ }^{21}$.

Tierra y, sobre todo, territorio son aquí conceptos claves, asociados a una comunidad social que tiene consciencia de sí misma, una conciencia que además es instrumentada políticamente. L'Etoile dice que este tipo de museos «han tenido históricamente un papel crucial en la producción de una conciencia de sí fundada en el sentimiento de la grandeza pasada» ${ }^{22}$. Una noción, muy familiar para todos, que está tomada directamente de Max Weber y su famoso ejemplo del museo alsaciano de Colmar ${ }^{23}$. Por este ejemplo queda claro que la «comunidad» de la que hablaba Lomnitz es aquí un «grupo étnico», fundamento de una «nacionalidad» (ambos términos según los emplea Weber), que legitima su afirmación identitaria y sus aspiraciones políticas, entre otras cosas, a partir de un pasado cargado de prestigio cultural, para lo cual el museo como institución, con su fondo patrimonial y su discurso expositivo, resulta un

20 «L'héritage de projects d'affirmation d'une identité collective enracinée dans un passé commun» (L'Etoile, 2007: 12).

21 «Nous incarné dans le musée par divers objets qui témoignent du passé de la communauté en question et qui, le plus souvent, proviennent de son territoire» (L'Etoile, 2007: 12).

22 «De tels musées ont historiquement joué un rôle crucial dans la production d'une conscience de soi fondée sur le sentiment de la grandeur passée» (L’Etoile, 2007: 12).

23 Weber, 1978: 396 (se trata del cap. V: «Ethnic Group», apart. 3: «Nationality and Cultural Prestige»). 
instrumento de afirmación, confirmación y difusión de primera magnitud. Estamos ante la definición misma del museo nacional - o del regional que aspira a nacional - en su versión más pura, la que explica por qué este tipo de instituciones parece ser uno de los requisitos imprescindibles a la hora de construir los estados-nacionales, al menos a lo largo del siglo XIX, a un lado y otro del Atlántico, y desde luego en Latinoamérica. Fenómeno confirmado por las experiencias asiáticas y africanas del siglo XX.

Frente a todo esto, que incluye museos nacionales, regionales, comunitarios y hasta de grupos profesionales, lo que L'Etoile llama «museos de los otros» (musées des Autres) resulta no ya una rareza, sino una auténtica aberración hermenéutica. Los «museos de los otros» literalmente no tendrían contenido propio, ni responderían a una pregunta o un objetivo precisos. Es más, la delimitación de sus contenidos solo puede hacerse de forma «negativa» ${ }^{24}$, porque remite a todo lo que se contrapone a nosotros, lo que no es de aquí, lo que no es propio. En esta forma de definir, por supuesto, está pesando una larga y bien conocida tradición francesa de reflexión sobre lo «exótico» y la pasión por el «exotismo» que, aunque sea muy atractiva literariamente, poco o nada ayuda a la cuestión que nos ocupa y sí, en cambio, la complica. Por lo que no vamos a tenerla en cuenta.

L'Etoile, por esta vía, se ha metido en un grave problema hermenéutico del que solo puede salir recurriendo a una línea de argumentación muy diferente. Es la que le proporcionan estudios sobre los museos antropológicos como los de Nélia Dias y George W. Stocking ${ }^{25}$, en los que este tipo de instituciones se presentan como fundamentales para el desarrollo y profesionalización de la Antropología en cuanto disciplina a lo largo del siglo XIX y parte del XX. Irónicamente, según L'Etoile sería la Antropología, con su «preocupación por identificar y clasificar esos objetos venidos de ultramar y, en general, por resolver los problemas intelectuales planteados por el encuentro entre mundos diferentes», la que finalmente llenaría de sentido a los «museos de los otros», haciendo posible que desde ellos se proponga «una respuesta a la pregunta de "quiénes son los otros" ${ }^{26}$.

Pero lo cierto es que esta solución parece bastante débil y resulta además insuficiente. Sobre todo si nuestro análisis no utiliza el caso francés —o más

24 «La délimitation du domaine du musée est alors négative» (L’Etoile, 2007: 12-13).

25 Véase especialmente Dias, 1991 y Stocking, 1985.

26 «C'est pour une large part dans les musées que l'anthropologie s'est constituée en discipline, au XIXe siècle, autour de la préocupation d'identifier et de classer ces objets venus d'ailleurs et, plus largement, de résoudre les problèmes intellectuels posés par la rencontre de mondes differents... Les musées consacrés à l'ethnographie... proposent une réponse à la question: “Qui sont les Autres”» (L'Etoile, 2007: 13). 
bien, el de París y sus museos- como único referente de estudio y si además entendemos que la dominación colonial no es ni ha sido la única que puede establecerse con los «otros».

Los planteamientos de L'Etoile son la última formulación de una idea que, de un modo u otro, suele asociarse desde hace mucho tiempo a la Antropología como disciplina y, en consecuencia, a «sus» museos. Pero hay buenas razones para preguntarse si es correcto o incluso útil seguir manteniendo una clasificación que habla de dos tipos de museos: museos de la comunidad o de la propia sociedad (que hablan de uno mismo), y museos de los otros (que no se sabe muy bien de qué hablan, pero que en todo caso se refieren a los demás, a los que no somos nosotros). Y suponer además que solo estos segundos son los «museos antropológicos» o, al menos, «etnológicos». En realidad, cabe preguntarse dónde queda la Antropología en semejante clasificación e, incluso, qué sentido tendría como disciplina fuera de su espacio académico en Europa (y los EE.UU.), actuando en los diferentes países de Latinoamérica, por ejemplo. Y qué pasa con los «otros» que están y forman parte del «nosotros», presentes de forma obvia en todas las sociedades multiétnicas del siglo XX y XXI, pero también en las sociedades europeas más homogéneas del siglo XIX y principios del XX, con sus diferentes poblaciones «tradicionales» o «rurales», a los que desde luego las diferentes disciplinas antropológicas siempre prestaron notable atención.

Ya en otro lugar he cuestionado la idea de que la Antropología es una disciplina construida exclusivamente «hacia afuera», desde lo colonial. Bien al contrario, la Antropología se institucionaliza y se financia en buena medida para resolver problemas «hacia adentro», los que afectan a la propia población nacional, respondiendo a las necesidades de su caracterización y definición, en relación estrecha con la construcción de los estados-nacionales. Aspecto esencial que explica su difusión e institucionalización no solo en Europa, sino también en Estados Unidos, Canadá y América Latina, aunque con características diferentes en cada caso, adaptadas a las necesidades de cada lugar ${ }^{27}$. Eso explica, por supuesto, que en México el museo nacional por antonomasia sea precisamente el Museo de Antropología, algo que se ajusta muy mal al modelo de «museo de los otros». Pero también tiene mucho que ver con la complicadísima trayectoria seguida por una institución como el Museo Nacional de Arqueología y Etnología de Guatemala, y el problema de cómo ese país centroamericano resolvía qué hacer con su propia población indígena. Por poner dos ejemplos magníficos que casi constituyen modelos contrapuestos.

27 Véase Bustamante, 2005. 
Benoît de l'Etoile ha reducido el complejo tema de la antropología y de sus museos exclusivamente a la cuestión identitaria. Y no hay nada más resbaladizo, además de cambiante y múltiple, que la identidad. Una noción que, para agravar aún más las cosas, es esencialmente «deíctica» (es decir, que solo puede definirse de forma referencial, como las nociones de «aquí» y «allí» o como las relaciones de parentesco, que solo pueden describirse en función de un «ego», por ejemplo).

Es cierto que la crisis que está afectando a los museos de contenidos antropológicos deriva en buena medida de conflictos que nada tienen que ver con cuestiones científicas propiamente dichas, sino con lo que podríamos llamar una "política de identidades». Las reformas que se están llevando a cabo en este tipo de museos, a las que aludíamos al comienzo de estas páginas, tienen mucho más que ver con un intento de evitar el conflicto político (o incluso con la aplicación, a veces puramente preventiva, de lo politically correct), que con un debate científico de cualquier tipo. De hecho algunos planteamientos críticos han llegado a contraponer lo científico y el cuerpo humano de tal manera (sobre todo en relación con el género o la raza), que de ese binomio solo parece poderse desprender una relación inherentemente incorrect, basada en la dominación y el abuso, cuando no en el exterminio genocida ${ }^{28}$. Esta tendencia viene a sumarse a otra más general que, desde la década de 1970, ha ido progresivamente cuestionando lo «científico», en sentido amplio, atacando su carácter «elitista», que lo haría sospechoso de ser asocial o incluso antisocial, y por utilizar prácticas de investigación y de demostración poco o nada democráticas, al ser difíciles de entender por el «gran público», que — sobre todo gracias a los medios de comunicación - pasa a identificarse como la «sociedad»y, consecuentemente, debe ser quien ejerza el control de sus actividades, fijando límites y hasta su conveniencia. Es decir, lo contrario de lo que

28 Este tipo de planteamientos extremos, de inspiración solo inicialmente foucaultiana, suelen ser muy bien intencionados pero producen graves distorsiones en situaciones que ya son suficientemente problemáticas de por sí. Hay múltiples casos que podrían servir de ejemplo, entre ellos las distintas contribuciones del Colectivo GUIAS (Grupo Universitario de Investigaciones en Antropología Social), especialmente 2010 y 2011, formado por Fernando Miguel Pepe, Miguel Añón Suárez y Patricio Harrison, que tiene como referente crítico el Museo de La Plata (provincia de Buenos Aires) y sus riquísimos fondos, entre otras cosas, de restos humanos. La identificación de las prácticas antropológicas del XIX y principios del XX con actitudes y políticas genocidas similares a las del Tercer Reich o a las de la última dictadura argentina y sus desaparecidos, no me parece que ayude demasiado a entender y valorar una institución como el Museo de La Plata (uno de los más modernos y completos del continente en su época), ni la labor de los estudiosos que lo crearon y trabajaron en él, como Francisco Moreno o Robert Lehmann-Nitsche, que merecen una consideración algo más sosegada y ecuánime. 
iba asociado a la vieja noción mertoniana de la Ciencia (en singular y con mayúscula $)^{29}$.

Pero, aunque los temas identitarios sean políticamente pertinentes, sigue sin tener sentido reducir los museos de contenidos antropológicos a aquellos que clasificamos como «museos de los otros», y más inadecuado aún me parece considerar que los museos de comunidad o de la propia sociedad («museos de uno mismo») no pueden ser manifiestamente antropológicos. Las comunidades indígenas de Oaxaca hacen sus propios museos, «museos de uno mismo» en su sentido más estricto, destinados a funcionar como referentes para la propia identidad, como lugares de la memoria y canon de prestigio, creados precisamente para referirse a unas prácticas y a un tipo de relaciones sociales que están desapareciendo o que ya lo han hecho, por la emigración que vacía los campos y/o por la entrada de nuevas prácticas y técnicas sustitutorias procedentes de los ámbitos urbanos. Se trata sin duda alguna de museos de comunidad, que además suelen ser comunitarios en su administración; pero al mismo tiempo son museos indudablemente antropológicos o etnológicos o de tradiciones populares o como se los quiera llamar.

Y el actual National Museum of the American Indian de la Smithsonian Institution, es un museo nacional, es un museo antropológico de referencia internacional y es además el museo de las comunidades indígenas norteamericanas, que son quienes deciden los criterios expositivos y el discurso empleado (origen de más de una crítica, como ya se ha comentado en otra parte). Y de este tipo existen muchos otros ejemplos en Estados Unidos, Canadá y Latinoamérica, e igualmente en el área del Pacífico, Nueva Zelanda y Australia en especial ${ }^{30}$.

La identidad o, mejor dicho, la alteridad no es lo que caracteriza este tipo de museos, como tampoco es lo que define lo antropológico. Sí es un material de predilección para la Antropología, y el primero que se le atribuyó y se sigue atribuyendo a la disciplina como campo de especialización profesional. Pero la Antropología en sus planteamientos, objetivos, métodos y sujetos de estudio aspira y ha aspirado siempre a la universalidad de lo humano, sin haber pretendido nunca reducirse a los que son más diferentes de nosotros o más supuestamente primitivos o salvajes. Esa universalidad de las propuestas y del discurso antropológico es la que le dio más fuerza en influencia social en el siglo XIX, momento clave de su profesionalización e institucionalización en el que los

29 Tomo esta última idea de Jansen, 2011.

${ }^{30}$ La literatura sobre este nuevo género de instituciones y los problemas que generan empieza a ser importante. Véase, por ejemplo, Jacknis, 2006. McCarthy, 2007. Sleeper-Smith (ed.), 2009. Barkan, Bush (eds.), 2002. Kreps, 2003. 
museos desempeñaron precisamente un papel determinante. Pero, además, es preciso recordar que su condición de disciplina científica no depende ni ha dependido nunca de la raza, la cultura o el género al que pertenezca la persona que la pone en práctica, que la ejercita en el campo, en el aula o en el laboratorio. De hecho, ya había personas procedentes de los lugares más exóticos formándose en L'École d'Anthropologie de París en la época de Paul Broca; o, por ejemplo, el reducido pero selecto grupo de antropólogos que trabajaba en el Bureau of American Ethnology de la Smithsonian Institution, al que debemos una formidable serie de monografías de referencia, incluyó desde el principio native americans. Una tendencia histórica que hoy se ha vuelto dominante: antropólogos con un origen étnico preciso, trabajando sobre su propia tradición cultural y lingüística. Comunidades aborígenes definiendo la forma en que desean verse y ser vistos, poniendo límites a lo que debe o puede ser conocido, según sus planteamientos, pero también haciendo posible otras perspectivas y otros campos de trabajo.

La cuestión identitaria resulta muy insuficiente para dar cuenta de la complejidad de la disciplina antropológica en la actualidad y la de sus museos. Hay otro tipo de planteamientos que tienen consecuencias muy profundas. Por ejemplo, el «horizonte» - universal, nacional o local_ desde el que trabaja el antropólogo, o a partir del cual se crea el museo, determina en cadena todo lo demás: desde la noción de patrimonio, pasando por el tipo de comunidad y hasta la propia finalidad de la institución o del trabajo antropológico. Precisamente una de las características que diferencian desde el siglo XIX las instituciones museísticas americanas - especialmente latinoamericanas - de las europeas, es precisamente el horizonte. Las europeas, quizá por ser algo más tempranas, solían tener una declarada vocación universalista; las americanas, quizá porque se concibieron como un elemento más del proceso de construcción nacional, pusieron como límite las fronteras del propio territorio o de la región en que se inscribían. Lo que resultó determinante para su desarrollo posterior, institucional y disciplinar.

El panorama latinoamericano (véanse los trabajos de Luis Gerardo Morales y Manuel Burón en este mismo número de la revista) nos muestra cómo, en la actualidad, domina cada vez más una tendencia que impone lo local, en un sentido mucho más reducido y fragmentario que el del horizonte «nacional», sobre lo general; lo que suele ir unido a una fuerte implicación de la comunidad social inmediata a la institución. Ambas cosas están incidiendo de forma importante en los modos tradicionales de legitimación, argumentación y comunicación utilizados por las disciplinas antropológicas y en los museos. Están afectando incluso a los protocolos habitualmente empleados por las disciplinas científicas, que son desechados, puestos en duda o directamente re- 
chazados a favor de otro tipo de planteamientos, digamos, no académicos y que abren magníficos espacios para la indagación antropológica. Si a todo ello le añadimos el debilitamiento generalizado de los museos nacionales en cuanto institución y todavía más del discurso que solían emplear desde el siglo XIX, ambas cosas como consecuencia de la crisis de prestigio y como modelo político del estado-nacional decimonónico (en el contexto de la globalización y la revalorización de minorías y grupos subalternos), se hace muy comprensible tanto la sorprendente eclosión de museos regionales, locales, subalternos, etc., como la tendencia de muchos museos nacionales a asumir el modelo estetizante, que hemos descrito más arriba, como una manera de evitar tensiones y aplazar conflictos. Pero ciertamente no de resolverlos, o de intentar superarlos.

Se trata de un universo fascinante, que se encuentra ahora mismo en pleno crecimiento y sujeto a profundas transformaciones. Un universo que muestra toda su riqueza y complejidad cuando se incorporan a su estudio los procesos que se están produciendo a ambos lados del Atlántico, aprovechado así la formidable batería experimental de casos que ofrece la América Latina.

\section{REFERENCIAS BIBLIOGRÁFICAS}

Barkan, Elazar y Bush, Ronald (eds.), Claiming the Stones, Naming the Bones. Cultural Property and the Negotiation of National and Ethnic Identity, Los Ángeles, Getty Research Institute, 2002.

Bustamante, Jesús, «La conformación de la antropología como disciplina científica, el Museo Nacional de México y los Congresos Internacionales de Americanistas», Revista de Indias, vol. LXV, núm. 234 (Madrid, 2005): 303-318.

Bustamante, Jesús, «La institucionalización de las ciencias antropológicas en las nuevas naciones y el papel de los museos», en Sandra Carreras y Katja Carrillo Zeiter (eds.), Las ciencias en la formación de la nación en América Latina (1810-1925), Berlín, Ibero-Amerikanisches Institut, en prensa.

Colectivo GUIAS, Antropología del genocidio. Identificación y restitución: «colecciones» de restos humanos en el Museo de La Plata, La Plata, De la Campana, 2010.

Colectivo GUIAS, El Familiar. Del ingenio La Esparanza al Museo de La Plata, La Plata, De la Campana, 2011.

Cooper, Karen Coody, Spirited encounters. American Indians protest museum policies and practices, Lanham (MD), Altamira Press, 2008.

Dias, Nélia, Le Musée d'Ethnographie du Trocadéro, 1879-1908: Anthropologie et Museologie en France, París, Éditions du CNRS, 1991. 
Dupaigne, Bernard, Le scandale des arts premiers. La véritable histoire du musée du quai Branly, París, Mille et une nuits, 2006.

Fabian, Johannes, Time and the Other. How Anthropology makes its Object, Nueva York, Columbia University Press, 1983.

Fabian, Johannes, Anthropology with an Attitude. Critical Essays, Stanford, Stanford University Press, 2001.

Fabian, Johannes, Memory against Culture. Arguments and Reminders, Durham, Duke University Press, 2007.

Jacknis, Ira, «A New Thing? The NMAI in Historical and Institutional Perspective», The American Indian Quaterly, vol. 30, núms. 3 y 4 (summer/fall 2006): 511-542.

Jansen, Axel, «Professionalization and Deprofessionalization in the Public Context of Science in the US and in Germany since 1970», presentación del proyecto (Volkswagen Stifttung) Professionalization and Deprofessionalization in the Public Context of Science since 1970. A Case-Based Analysis of Four Research Fields in the U.S. and in Germany, Madrid, Centro de Ciencias Humanas y Sociales, CSIC, 2011 (comunicación oral y ms.).

Karp, Ivan; Kreamer, Christine Mullen y Lavine, Steven D. (eds.), Museums and Communities: The Politics of Public Culture, Washington D.C., Smithsonian Institution Press, 1992.

Kreps, Christina F., Liberating Culture: Cross-cultural Perspectives on Museums, Curation, and Heritage Preservation, Londres, Routledge, 2003.

L'Etoile, Benoît de, Le goût des autres: De l'Exposition coloniale aux Arts premiers, París, Flammarion, 2007.

Lomnitz, Claudio, Modernidad indiana. Nueve ensayos sobre nación y mediación en México, México, Planeta, 1999.

Lonetree, Amy y Cobb, Amanda J. (eds.), The National Museum of the American Indian. Critical Conversations, Lincoln, University of Nebraska Press, 2008.

McCarthy, Conal, Exhibiting Maori. A History of Colonial Cultures of Display, Oxford, Berg Publisher, 2007

Peers, Laura y Brown, Alison Kay (eds.), Museums and Source Communities. A Routledge Reader, Londres, Routledge, 2003.

Price, Sally, Paris Primitive. Jacques Chirac's Museum on the Quai Branly, Chicago, Chicago University Press, 2007.

Rothstein, Edward, «Museum Review; Museum with an American Indian Voice», The New York Times (21 de septiembre de 2004). Edición digital: http://query.nytimes. $\mathrm{com} / \mathrm{gst} /$ fullpage.html?res=9E01E4D71539F932A1575AC0A9629C8B63

Sleeper-Smith, Susan (ed.), Contesting Knowledge. Museums and the Indigenous Perspectives, Lincoln, University of Nebraska Press, 2009. 
Stocking, George W. (ed.), Objects and Others: Essays on Museum and Material Culture, Madison, University of Wisconsin Press (Col. «History of Anthropology», vol. 3), 1985.

Taylor, Kate, «The Thorny Path to a National Black Museum», The New York Times (22 enero 2011). Edición digital: http://www.nytimes.com/2011/01/23/us/ 23smithsonian.html? $\mathrm{r}=1$

Weber, Max, Economy and Society. An Outline of Interpretative Sociology, edited by Guenther Roth and Claus Wittich, Berkeley, University of California Press, 1978.

West, W. Richard et al., The Changing Presentation of the American Indian: Museum and Native Cultures, Seattle and Washington, University of Washington Press and National Museum of the American Indian, Smithsonian Institution, 2000.

Fecha de recepción: 13 de septiembre de 2011.

Fecha de aceptación: 21 de diciembre de 2011.

\section{Museums, memory and anthropology on both sides of the Atlantic. Institutional crisis, nation building and the memory of colonization}

Museums of an anthropological nature, and/or those which refer to areas subjected to Western colonization, are currently undergoing a long crisis that is leading to a global renewal of the oldest institutions, the development of new museological models and the emergence of diverse critical and exhibition proposals. This affects anthropology as a scientific discipline but also has an increasingly significant effect on both history and the fine arts. This paper attempts to provide an overview of these developments based on a comparison of processes taking place on both sides of the Atlantic, and stressing the importance of Latin American cases because of their variety and originality, as well as the complexity of the socio-cultural situations with which they are associated.

KeY Words: Museums; memory; anthropology; colonization; nation building; patrimony; community; museological models. 